\title{
ECONOMÍA, EDUCACION Y EMIGRACION EN EL CHOCO: INFORME DE UN FUNCIONARIO DEL AMERICAN UNIVERSITIES FIELD STAFF
}

Thomas G. Sanders

\section{Introducción}

El departamento del Chocó es una región periférica dentro de una sociedad que se desarrolla alrededor de centros dinámicos como Bogotá, Cali y Medellín. En sí mismo, tiene casi todas las características atribuidas a las naciones atrasadas: un alto porcentaje de su población se ocupa en agricultura de subsistencia, dependencia de la "exportación" de productos primarios, potencia y medios de transporte inadecuados, la casi evidente falta de industria, la inhospitalidad geográfica y condiciones climáticas, y la pobreza extrema que se refleja en la falta de ahorro interno y de inversión. Determinantes comunes del nivel de desarrollo - como el ingreso nacional percápita - oscurecen las diferencias entre regiones como el Chocó, donde el standard de vida se compara con los menos desarrollados del mundo, y de los centros industriales urbanos modernos, muchos de cuyos ciudadanos viven de acuerdo con un patrón europeo o americano.

A medida que Colombia crece, ¿qué sucederá con el Chocó? Esta pregunta llega a ser especialmente picante porque, como indicaba en un artículo anterior ${ }^{7}$, ser chocoano no es solamente una denominación geográfica sino una conciencia racial y cultural. Muchos chocoanos se sienten ligados más estrechamente a gente con antecedentes raciales similares en el litoral pacífico, que se extiende hasta Panamá y Ecuador, que lo que se sienten con otros colombianos. Esta actitud es especialmente marcada en pueblos pequeños a lo largo de la Costa, en donde las únicas comunicaciones se hacen hacia el norte y el sur por mar (allí no hay carreteras hacia el interior), y la mayoría de las mercancías manufacturadas llegan como contrabando de Panamá a la cual es más fácil llegar que a cualquiera de las grandes ciudades colombianas.

Sin duda, el Chocó tiene recursos naturales para desarrollar. Sus habitantes hablan enardecidamente más que con optimismo de las "riquezas" de su departamento, pero no saben cómo utilizarlas. Asumiendo que la inversión y las habilidades foráneas serán atraídas hacia polos ya existentes de desarrollo en Colombia, el Chocó podría tratar de mantener su juventud dentro de la región dirigiéndola a técnicas relevantes para el desarrollo local. Desafortunadamente esto no ocurre, y la falla puede encontrarse básicamente en la orientación del sistema educativo. Mientras que la economía chocoana continúa a un nivel primitivo e improductivo, relegando la mayor parte de la población a la desnutrición, su juventud ambiciosa y educada vuelve la espalda, a menudo con pesar, para aplicar sus talentos en cualquiera otra parte.

La urbanización en América Latina se asocia con la modernización y el crecimiento de la industria, y como tal, ha estimulado una extensa literatura. Relativamente, se ha prestado menos atención a las áreas de las cuales vienen los migrantes. A menudo se arguye que el sistema de grandes o pequeñas propiedades rurales no proporciona trabajo a la creciente población rural. El Chocó tiene un alto porcentaje de emigración, pero su sistema de tenencia de la tierra no es ni latifundista ni minifundista. Su problema parece

\footnotetext{
7 Thomas G. Sanders Los negros del Chocó", (TGS-I-70) Fieldstaff Reports, West Coast South America Series, Vol. XVII, No. 2, 1970.
} 
ser de alguna manera diferente: el atraso de la región ofrece poca oportunidad para vivir decorosamente y el sistema de educación no entrena a la juventud para desarrollar los recursos disponibles. En efecto, la mayor demanda de habilidades de jóvenes chocoanos se encuentra más frecuentemente en otros departamentos de Colombia.

\section{Economía del Chocó}

El soporte de la economía chocoana ha sido siempre la minería: en el período colonial el oro y la plata, y hoy día el oro y el platino. Cerca del $25 \%$ de la población trabajadora son mineros quienes se concentran en pueblos pequeños en el Valle del río San Juan, tales como Condoto, Istmina y Nóvita.

El método predominante, el mazamorreo, no ha cambiado desde los tiempos coloniales. Algo del sabor de la vida de minero se puede extractar de la siguiente autobiografía que me relaté una mujer de Condoto:

"Mi nombre es Carmen. Nací en Condoto. Mi padre Eliécer y mi madre Dominga, trabajaron en minería y comercio (esto consiste en el cambio de oro en Quibdo por pescado y otros artículos de consumo). Mi padre fue un trabajador inteligente, estimado por todos y que ayudaba a los necesitados. Era alto, delgado y de color moreno. Vivió con alguna comodidad; no fue pobre.

Cuando yo tenía siete años, empecé a trabajar jugando en la minería. Mis hermanos y hermanas también trabajaban. En las minas extraíamos el oro y el platino, seis días a la semana, de siete de la mañana a seis de la tarde; excepto en los períodos de descanso. El domingo íbamos a misa, bailábamos y hacíamos comida especial. Los hombres y las mujeres tomaban aguardiente los domingos, los días de fiesta y durante las lluvias. En las minas trabajábamos con las manos, con bateas (tazones pandos de madera), picas y palas. La arcilla está allá y con la pica y la pala se afloja y se saca, y después se bota a un lado. Se pone la arcilla en la batea y se mezcla con agua. Esto da un balasto de piedras, arena, platino, oro y jagua (arena negra). Se le da vueltas a esto y se limpia de piedras y arena. Enseguida se separa el platino del oro y la jagua, con un magneto, y después se separa el oro de la jagua con la mano. Se seca y se vende".

Toda la población de los pueblos como Condoto se ocupa de la minería y de las actividades comerciales que dependen de ella. El oro y el platino se venden a través de dos medios: El Banco de la República o comerciantes individuales. El Banco paga un mejor precio, pero los comerciantes lo compensan dando crédito a los mineros. Todas las firmas comerciales, de la principal y única calle de Condoto, tienen pequeñas balanzas para pesar los metales, lo que usualmente se hace en castellanos (cerca de la sexta parte de una onza). Generalmente todo el oro termina en el Banco, puesto que el Gobierno tiene el monopolio de esta mercancía.

Si el oro de mazamorreo y el platino, que es más valioso, dan una imagen de prosperidad, ésta se disipa rápidamente con una visita a uno de los pueblos mineros. La producción por individuo es muy baja. Los mineros se alimentan tan mal como otros chocoanos y las casas son como cualquiera otra del Chocó, hechas de madera con techos de paja o lata, con muy pocos muebles y ninguna comodidad.

La única industria altamente técnica en el Chocó es la Compañía Minera ChocóPacífico de Andagoya, filial de la Corporación Minera Internacional de New York. La Chocó-Pacífico opera cuatro dragas las cuales produjeron cerca de 25.000 onzas de oro y 14.000 onzas de platino el año pasado (1969). Las dragas se desplazan lentamente a lo 
largo de los bancos de los ríos u otros depósitos de agua y con un cinturón de baldes de hierro sacan y procesan grandes cantidades de suelo que contiene el metal.

Las casas pulcras y sólidas de la compañía en Andagoya contrastan notoriamente con las casas de los pueblos mineros pequeños. Es de conocimiento general que la compañía paga a sus trabajadores y a sus empleados de cuello blanco los mejores salarios en el Chocó: un trabajador ordinario recibe alrededor de dos dólares con cincuenta al día, junto con extras de mayor valor aún, tales como vivienda gratuita, colegios, atención médica, luz y agua. Además hay bonificaciones en navidad y días de fiesta, generosas vacaciones, retiro con pensión a la edad de 55 años, y el derecho de comprar alimentos en el almacén de la compañía a precios rebajados. A los trabajadores de cuello blanco se les paga aún mejor. Estos beneficios se adquieren en su mayor parte por las presiones del sindicato, que es la organización de trabajadores más efectiva del Chocó.

La Compañía ha tratado de estar de acuerdo con los rasgos distintivos de la cultura chocoana. Por ejemplo, da subsidio a los hijos reconocidos por sus trabajadores aunque sean hijos de distintas mujeres. En la comunidad de Andagoyita, al otro lado del río Condoto, la compañía construyó escuelas e iglesia porque sabe que casi toda la población está compuesta de familiares de sus trabajadores. En contraste con una generación anterior, cuando los negros tenían solamente trabajos serviles, la Compañía ha tratado de darle a los negros puestos de responsabilidad. Esto no ha sido fácil, pues, como me dijo el superintendente, a los negros no les gusta la carga de ejercer autoridad sobre su propia gente. Realmente el único funcionario negro, con más alto cargo en la Compañía, es el médico director del hospital.

Muchos nacionalistas jóvenes chocoanos y colombianos, ven la Chocó-Pacífico como un enclave del imperialismo americano que logra ampliar ganancias extractando riquezas irrecuperables de la región. En realidad, la producción de la compañía es la mitad de lo que fue hace veinte años, y la Compañía trató de venderse en una ocasión, sin éxito, al Gobierno Colombiano. De los 500 trabajadores, menos de 20 son extranjeros, casi todos antiguos residentes quienes permanecen porque les gusta el Chocó y tienen familias allí. El último americano que trabajó en la compañía se jubiló recientemente y se quedó en la costa del Chocó.

Aunque los pequeños mineros producen la mayor parte del oro y del platino del Chocó, el rendimiento individual es pequeño a causa de la tecnología primitiva. El atraso de los métodos comunes me fue revelado en una conversación con funcionarios del Ministerio de Minas y Petróleo cuando hablamos acerca de las medidas del gobierno para introducir avances técnicos entre los pequeños mineros. El Ministerio arrienda motobombas para sacar el agua de las áreas de mazamorreo y está tratando de introducir el uso de la artesa oscilante ("California Cradle",). Cuando pregunté qué era esto, un funcionario nos señalé un cuadro en la pared representando una escena familiar de los años cuarenta y nueve ${ }^{8}$, que mostraba un minero barbado de pie junto a un dique de madera en cuyo extremo superior se pasa y se procesa la arena mojada. En la pequeña minería chocoana, una "mejora técnica" es la adopción de un artefacto que se usó en Estados Unidos hace más de un siglo. Sorprendentemente, no obstante, pocos mineros hacen uso de estas innovaciones. La tradición del mazamorreo individual y familiar, no solo es una destreza sino una fuente de orgullo que cambia lentamente. Organizar un grupo de gente e invertir

8 "Forty-miners": Personas que fueron a California en 1849 durante la fiebre del oro (Nota de las traductoras). 
en maquinaria, o aún la noción de ganar más que para la mera subsistencia es a menudo una forma extraña de pensar para los mineros chocoanos.

Si la minería es la base de la exportación del Chocó, la agricultura de subsistencia es la vida de casi la mitad de la población. Los productos —arroz, maíz, banano, yuca y frutas - proveen una dieta inadecuada y monótona. Además, a menudo las cosechas se destruyen por el desbordamiento de los ríos, los medios de transporte al pie de los cuales vive casi toda la población rural.

El Gobierno Colombiano tiene instituciones que ofrecen crédito a pequeños agricultores, pero la mayoría de los chocoanos no reúnen los requisitos dado que muy pocos de ellos tienen escritura que los acredite como dueños de la tierra. La propiedad como tal, con base legal, nunca fue parte de su cultura. Con abundancia de tierra, el agricultor simplemente cultivaba cualquier parcela que encontraba libre. Como ha ocurrido con los reclamos sobre la minería, esto nunca causó problemas, porque el mutuo respeto más que la Ley, garantizaban sus derechos. El Instituto de la Reforma Agraria del Gobierno, ha estado tratando de aclarar los títulos de la tierra en el Chocó, empezando con la Costa Atlántica y el Río Atrato, pero, puesto que este proyecto no ha llegado a la mayor parte del Chocó, el crédito actual se da predominantemente a individuos, a menudo foráneos, cuya mentalidad capitalista los condujo a ganar y a verificar la posesión de su tierra. No obstante, el Chocó es considerado como uno de los ejemplos de mayor éxito en la actividad de la Reforma Agraria Colombiana.

Un programa de mayor impacto en las áreas de Quibdo-Istmina, esta conformado por una serie de proyectos experimentales bajo los auspicios del Instituto Colombiano de Agricultura y Ganadería, con la asistencia financiera de la FAO (Organización para la Agricultura y Alimentación). En realidad, los expertos no saben qué puede cultivarse con éxito, no solamente para mejorar la dieta local, sino también para exportar, dado que el Chocó experimenta lluvias torrenciales que no son comparables a ninguna otra región del hemisferio occidental. En algunas comunidades se están experimentando nuevas variedades de arroz, caña de azúcar, y yuca, uso de matamalezas, mayor densidad de cultivos y la introducción de nuevas frutas y verduras. Pero la experimentación de la ganadería está actualmente restringida a causa de que el Instituto no tiene un veterinario, y no hay más de cuatro agrónomos en el Chocó.

Aunque la agricultura marcha bien, la región presenta otros obstáculos. En el mercado de Quibdo se pueden comprar piñas enormemente grandes, baratas y sabrosas. Un día me presentaron al señor Aladino Palacios, un empresario negro quien cultiva la mayor parte de ellas. Me dijo que tenía 40.000 matas de piña en Villa Conto, a una hora en lancha de Quibdó, que producen mucho más de lo que él puede vender. El problema es de transporte. Villa Conto es accesible solamente por vía fluvial: Para extender su mercado de Quibdó a Medelín, debe transportar las piñas por lancha a un camión y enviarlas en una jornada de un día, y hasta el momento no ha podido resolver allá lo concerniente al mercado.

El transporte es también un problema serio para la producción de madera, otro recurso chocoano importante. Como en muchas regiones de la selva, el Chocó posee una rica variedad de árboles, que hasta ahora ha sido explotada a lo largo de las pocas carreteras y los numerosos ríos por los cuales se puede transportar a otros sitios. En el interior del departamento la tecnología maderera no ha progresado lo suficiente, aun se utiliza el servicio de mulas. Usualmente un individuo rema en su piragua hasta que encuentre un árbol que pueda tumular dentro del río y flote hasta la carretera o el aserradero más cercano. Me dicen que en Juradó, un pueblo aislado en la Costa del Pacífico cerca de la 
frontera panameña con recursos madereros especialmente finos, el corte es más avanzado. Pero generalmente, allí y en la costa, los chocoanos se sienten engañados por los intermediarios foráneos quienes les pagan precios mínimos y se apropian de enormes ganancias por transportar la madera hacia el sur, al puerto de Buenaventura.

Probablemente en el Chocó, el elemento más prometedor y menos desarrollado es la pesca. Contrariamente al Perú y a Chile, Colombia ha explotado muy poco su costa pacífica, la mitad de la cual se encuentra en el Chocó. En efecto, la costa chocoana es casi inhabitada exceptuando unos pocos pueblos, uno de los cuales solamente, Bahía Solano, tiene mercado externo para el pescado. Esta actividad económica también es primitiva. Los pescadores usan pequeños botes hechos de troncos ahuecados a cuyos lados colocan tablones para profundizarlos y la potencia es dada por un motor fuera de borda. Bahía Solano tiene facilidades atorgadas a través de una agencia gubernamental para preservar el pescado hasta que pueda llevarse a Medellín por transporte aéreo diario. En los mejores meses salen así 30.000 libras, pero la mayor parte del precio de venta pasa a los transportadores e intermediarios más que a los pescadores chocoanos (en Bahía Solano compré por un dólar un pámpano de 18 libras; en Quibdó el precio del "pescado de mar" es de un dólar por kilo). Los ciudadanos de Bahía Solano creen que la pesca tiene un porvenir brillante, una vez que la carretera que ahora está en construcción conecte a su pueblo con el interior cerca de Istmina, y el pescado se pueda transportar de allí a Medellín. Sin embargo, no hay señales de un esfuerzo público o privado para introducir métodos más sofisticados para la pesca.

Finalmente, merece mención la industria regional de la empresa de licores del Chocó. Cada Departamento en Colombia tiene el monopolio de la producción de bebidas alcohólicas, con mercado nacional según convenios con otros departamentos. La empresa de Licores del Chocó no tenía una historia sobresaliente al producir aguardiente de baja calidad, hasta 1963, cuando invitó a una firma francesa, Le Paige and Urbain, para mejorar su producto. El resultado fue una fórmula secreta y distinta para el aguardiente y el brandy de cerezas, los cuales ganaron los primeros premios en un concurso suramericano. La empresa también produce ginebra, ron y triple sec, pero el aguardiente anisado suave "Platino" es su orgullo. La empresa es aún pequeña; emplea 34 personas en un edificio de concreto fuera de Quibdó que produce 40.000 litros al mes. Pero los chocoanos ven un futuro en ésta, su única "fábrica". En Bogotá, las cigarrerías anuncian ahora "Platino" con grandes avisos, y durante el año pasado se exhibió en las Ferias de Alemania Oriental y España.

\section{Programas de desarrollo}

De estas actividades sencillas, junto con el comercio, la administración pública y la educación, los 200.000 chocoanos derivan un vivir incierto y parco. Chocó, a diferencia de los Llanos Orientales y de la selva al sur de Colombia, no proyecta una imagen de oportunidad. Su población se estableció hace tiempo; sus productos básicos se conocen y todo el mundo sabe que los chocoanos son pobres. Individuos emprendedores del Departamento vecino de Antioquia entran al Chocó en pequeño número, pero ellos mismos son usualmente pobres y su capital solamente les permite realizar actividades en pequeña escala como el comercio, el aserrío o una máquina para limpiar el arroz. Con una población pequeña y dispersa y la falta de carreteras, el Chocó no puede más que sostener la industria local más sencilla (se habla de establecer una fábrica de gaseosas). Por consiguiente, el desarrollo económico debe expandirse, diversificarse, modernizarse y hallar nuevos mercados para las actividades existentes. 
El gobierno colombiano no ha ignorado al Chocó: en los años 50 hizo un análisis detallado de los recursos humanos y naturales de la región, que produjo numerosas proposiciones para incrementar y mejorar la producción y adjudicó una partida al gobierno departamental para fines de desarrollo. Durante la década pasada, parte de este dinero se destiné a nuevas escuelas, al mejoramiento de centros de salud, de energía eléctrica y especialmente para carreteras cortas; pero todo esto solamente mellé las necesidades infraestructurales de la región. Muchos de los fondos en vez de ser racionalmente asignados para el desarrollo, se malgastaron o se emplearon en pagar sueldos atrasados de los maestros, mientras que las presiones políticas, más que un plan total de comunicaciones, determinaba usualmente las prioridades de construcción de carreteras. Puesto que la presión para empleos públicos es tan grande en el Chocó, una cantidad considerable también fue para "administración", esto es, numerosos supervisores, asistentes, secretarias y recepcionistas de pequeños proyectos. Una de las personas más francas, a quien conocí en el Chocó fue el joven y dinámico Director de Planeación, quien había dejado un buen trabajo como ingeniero civil cerca de Bogotá, para regresar y ayudar a su región. Según sus propias palabras "no hay casi planeación en el Departamento de Planeación; este es solamente un canal para mantener fondos de varios proyectos que se han constituido por una variedad de razones sin integración alguna". El ha estado trabajando por algunos meses, simplemente para averiguar dónde se ha ido el dinero de todos estos años, y una vez que lo supo, trató de renunciar.

Muchos chocoanos esperan como tabla de salvación, la terminación de la Carretera Panamericana o la construcción de un canal que pase por el departamento y una los Océanos Atlántico y Pacífico. La única sección de la Carretera Panamericana que está incompleta es la de la parte sur de Panamá y Colombia. Se ha proyectado que vaya desde la frontera panameña a lo largo de la costa chocoana hasta Bahía Solano, virando luego hacia el Sur-Oriente, cerca de Istmina y pase al Departamento de Risaralda. Se están haciendo algunas mediciones topográficas y algo de construcción, pero a ese ritmo se prolongará por décadas. Ni siquiera el gobierno Colombiano muestra mucho interés en acelerar el proyecto. De acuerdo con la opinión de un ciudadano de Bahía Solano, "la carretera es esencial para el desarrollo del Chocó, pero no podemos esperarla". El chocó figura también en algunas propuestas para un canal, la más seria y relativamente barata es la de usar el Río Atrato para entrar del Atlántico y salir al Pacífico en Panamá. Esta propuesta transformaría indudablemente la parte norte del Chocó, pero nadie puede garantizar que un futuro canal pasará por Colombia y no por algunas rutas alternativas.

Mientras tanto, el Chocó mismo continúa desintegrado y la falta de medios de comunicación con otros sitios ahuyenta el capital necesario para el desarrollo. Los habitantes de Bahía Solano reclaman que tienen el mejor puerto potencial de Colombia, pero ¿qué tan útil es un puerto sin carreteras de acceso?, ¿quién construiría una fábrica en Quibdó, cuando para ir a Medellín, el mercado razonable más cercano, se necesita un día completo de viaje a través de una carretera angosta y empolvada? El Chocó se divide en regiones que difieren en actividad y orientación pero que están unidas por el sentimiento común de ser chocoanas y olvidadas. La Costa del Caribe y el Bajo Atrato, con un clima más seco y un potencial considerable de agricultura, se unen a ciudades porteñas como Turbo, Barranquilla y Cartagena. Quibdó es un centro administrativo y comercial que sirve al Alto Atrato y se orienta a Medellín. El área minera de San Juan vacila entre Quibdó, Medellín (por carretera) y Buenaventura (por vía fluvial). Los pueblos costeros dependen de Buenaventura y Panamá, puesto que solamente se unen con el interior por vía aérea de Bahía Solano a Quibdó. 


\section{Función de la educación en el Chocó}

Solamente en un factor conducente al desarrollo - la educación - el Chocó se levanta
con fuerza. El optimismo acerca del valor de la educación se ha difundido ampliamente, aún entre los elementos más pobres de la población y está simbolizado por un lema en un edificio escolar fuera de Quibdó: "Estudiando más transformaremos a Colombia". Antes de los exámenes finales muchos jóvenes se pasean por las calles a la una o dos de la mañana, tratando de concentrarse en sus libros. En los pueblos todos los niños asisten a la escuela primaria (gratuita) y en Quibdó, por lo menos, la mayoría continúa con la escuela secundaria con grandes sacrificios financieros de parte de sus padres. En las áreas rurales más sencillas hay escuelas, usualmente de un salón y construida de la misma madera y paja que las casas del vecindario.

Es difícil obtener cifras exactas de los niveles de educación chocoanos. Según las cifras del plan de desarrollo regional de 1951, el $72.7 \%$ del total de la población era analfabeta; el $37.4 \%$ en los pueblos principales. Desde entonces, se han construido muchas escuelas, se intensificaron las demandas para cargos docentes, y lo que es especialmente importante, aumentaron las oportunidades educativas para las personas de color. Funcionarios de la Secretaría de Educación de Quibdó, arguyen que el analfabetismo es ahora uno de los más bajos en Colombia, de solamente $15 \%$, y que se encuentra principalmente en las personas de edad y en las áreas rurales aisladas. El departamento tiene 415 escuelas primarias de las cuales 62 son urbanas y el resto rurales. Hay 33 escuelas secundarias, 17 oficiales y 16 bajo la dirección de la Iglesia Católica. Todas las escuelas secundarias cobran pensión, siendo la mínima lo equivalente a un dólar mensual. La mayoría ofrece solamente 4 años del ciclo de 6 años, pero los estudiantes que desean terminar el bachillerato (escuela secundaría) u obtener un certificado de enseñanza se trasladan a otras instituciones. En consecuencia, muchos alumnos de Quibdó vienen de comunidades más pequeñas con pocas facilidades educacionales para aprovechar las escuelas de la capital del departamento.

La intensa preocupación por la educación hace del Chocó un lugar especialmente apropiado para lanzar una pregunta relevante para muchas partes de Latinoamérica: ¿educación para qué? O más concretamente: ¿contribuye la educación al desarrollo?

El currículum del Chocó está de acuerdo con un patrón emanado de Bogotá y usualmente llamado "clásico". El producto esperado es una persona con cultura". Desde los primeros años el alumno asciende a través de un cubrimiento gradual y extenso a lo que se considera el conocimiento universal: matemática, idiomas (desde el español hasta los idiomas extranjeros empezando con el inglés), geografía, ciencias biológicas, ciencias físicas, educación cívica y "urbanidad" (comportamiento apropiado llamado algunas veces "cultura") y religión. En historia y geografía se parte del estudio de Colombia siguiendo luego con Latinoamérica y otros Continentes. Las clases de ciencias no tiene laboratorios ni experimentos; en otros campos se confían a la memoria de hechos establecidos. Mientras en teoría el currículo se puede modificar para adaptarlo a las necesidades locales, en la práctica no se hace nada al respecto. De ahí que las ciencias biológicas no enseñan al chocoano acerca de su flora y fauna, ni la educación cívica le da información sobre los problemas y la política del Chocó. Mientras que este enfoque no es desconocido en Estados Unidos, en Colombia asume una forma especialmente rígida y esterotipada. En el Chocó tiene como efecto que el estudiante memorice los hechos sobre fenómenos universales, más que confrontarlos con los problemas locales que se reflejen en su solución. 
El nivel secundario amplía lo que se aprendió en la escuela primaria. El estudiante puede escoger entre un instituto pedagógico ("Normales"), que lo capacitará para enseñar, y un entrenamiento pre-universitario que lo conduce al bachillerato. Cuatro pueblos pequeños tienen institutos agrícolas, de menor prestigio, que dan un título de "Experto en Agricultura y Ganadería" después de cuatro años de estudio. ¿Qué sucede con los que se gradúan en la escuela secundaria? Unos enseñan, otros trabajan en la congestionada administración pública y otros consiguen trabajo en bancos o firmas comerciales. Irónicamente, lo único que en realidad aprenden es a enseñar, puesto que no existe la instrucción en administración pública o comercial.

Uno de los pocos chocoanos consciente de que la educación es un problema, siendo él mismo un profesor, comenta: "En la primaria los niños aprenden por lo menos algo útil: a leer y escribir. La secundaria es una completa pérdida de tiempo". ¿Por qué? Los institutos de pedagogía normalmente colocarían a sus graduandos en el sistema escolar en expansión del departamento, pero desde hace algún tiempo la oferta de profesores ha excedido la demanda. Comúnmente se dice que la principal exportación del Chocó es la de profesores. De manera similar el bachillerato provee un entrenamiento pre-universitario que pocos chocoanos pueden permitirse ya que el asistir a la Universidad exige vivir fuera de la región. Uno escucha a menudo que el Chocó necesita una universidad, pero tal comentario refleja más el deseo y prestigio de la educación, que su pertinencia ya que pocos chocoanos graduados de la universidad regresan. Un joven me conté que de 30 chocoanos que asistían a la Universidad Nacional de Bogotá con él, solamente él y otro regresaron y ello por razones familiares. Un porcentaje considerable de los que regresan es de abogados, cuya vocación real es la de aprovechar la pequeña votación del Chocó para ganarse un puesto político.

La tendencia a emigrar de los chocoanos probablemente se intensificará muchísimo en las décadas venideras, especialmente entre los contingentes crecientes con educación secundaria y superior. La capacidad de las escuelas y la administración pública para proveer salarios y estatus de clase media para la gente de color con el necesario nivel educativo es limitada. La perspectiva de hacerse profesor o trabajar con el gobierno, motivé por mucho tiempo a las personas del Chocó hasta el punto de hacer sacrificios enormes por la educación como única manera de escapar de la pobreza en que la mayoría de ellos vivía; pero ahora se necesita influencia "Política" para conseguir un empleo, y los jóvenes asumen automáticamente que tendrán que encontrar trabajo en cualquiera otra parte. Entre 1951 y 1964, emigraron cerca de 20.000 chocoanos (una décima parte de la población), dejando al Chocó con el índice más bajo de crecimiento de población en Colombia. Puesto que no hay datos disponibles más recientes probablemente este índice es más alto ahora y aumentará en el futuro.

El nexo entre economía, educación y emigración es claro entonces. En una región de pobreza generalizada, habitada por descendientes de esclavos, la apertura de oportunidades de trabajo de clase media en la administración y la docencia, estimulaba las aspiraciones de educación, pero en ausencia del desarrollo económico que proveerá una amplia demanda en estos campos, la generación más joven debe salir a encontrar trabajo.

El énfasis de los chocoanos por la educación no se debe solamente a causas económicas. Es inexplicable si no se hace también referencia a la naturaleza de la discriminación racial en Colombia. Como lo anoté en un artículo anterior ${ }^{9}$, los chocoanos

\footnotetext{
${ }^{9}$ Ver nota No. 1 (N. de los trad.).

Digitalizado por RED ACADEMICA
} 
de color son víctimas de estereotipos raciales mantenidos comúnmente por los blancos. Dentro de la cultura negra, un hombre pobre y sin educación que sostiene su familia, o familias, y negocia honestamente con sus vecinos, se gana el respeto de ellos. Si tiene educación y "cultura" y vive de un salario de clase media le da aún más prestigio; pero su valor como hombre no depende de esto. ¿Qué puede decirse sobre sus relaciones con los blancos? Su color ordinariamente lo hace objeto de condescendencia estereotipada (perezoso, irresponsable), pero puede superar esto por otros haberes. Si es ducado y un "profesional", ordinariamente se le juzga como tal, en vez de juzgársele por los estereotipos raciales. Puede hacer frente a algunos obstáculos, pero generalmente se le da la aceptación y el prestigio apropiado a su posición. Por otra parte, la persona de color, carente de educación probablemente se juzga más por la imagen que los blancos tienen de los de color, que en términos de sus cualidades como hombre. Entonces, la educación llega a ser el recurso básico de los haberes ("cultura", forma de vida de clase media, estatus profesional y estabilidad laboral) por los cuales una persona de color puede lograr respeto no solamente entre los blancos del Chocó, sino también, si tienen que organizar su vida en el mundo exterior predominantemente blanco.

Los factores raciales también ayudan a determinar hacia dónde emigrará. Cali es la preferencia más común, especialmente entre los chocoanos educados, porque dada su población sustancialmente negra y mulata, ya se han abierto paso en la enseñanza y otras ocupaciones de clase media. Bogotá, es también atractiva porque las personas de color son tan escasas que no se han desarrollado estereotipos bien marcados. Medellín es la menos deseable porque sus estereotipos tienen raíces más profundas; pero aún allí se advierte que una persona de color con educación y buen desempeño laboral, gana aceptación. Los chocoanos tienen fama de adaptarse a nuevos ambientes, pero ellos saben muy bien la recepción que probablemente tendrán una vez salgan del Chocó.

El mismo sistema educativo que provee a los chocoanos de canales de movilidad ascendente, más que acercarlos, los aleja de la difícil tarea de desarrollar su propia región. No solamente no les enseña nada de minería, pesca, agricultura o de la explotación de bosques maderables, sino que, como en el resto de Latinoamérica, también los conduce hacia una actitud despreciativa por estas actividades. El hombre de "cultura" no se mezcla con las máquinas, con el trabajo manual o con actividades prácticas. Tampoco el mundo casi pre-capitalista del Chocó provee lo que los antioqueños derivan de la suya: una capacidad de organización, ahorro y proeza económica.

Es verdad que en forma limitada e incipiente el Chocó tiene ahora algunas formas prácticas de educación: los cuatro institutos agrícolas y cursos de agricultura, ganadería y explotación de maderas, organizados por el Gobierno a través de programas de extensión. Pero están dirigidas especialmente a personas que ya trabajan en estos campos, y lo que es más importante, les falta prestigio y articulación con el sistema educativo prevaleciente.

El rápido crecimiento de las ciudades de Latinoamérica a menudo se contempla como un mal a causa de los tugurios y el desempleo creado por los migrantes. Análisis más recientes enfatizan los beneficios, uno de los cuales es el traslado de la juventud provincial más educada y ambiciosa a los centros de desarrollo. Podemos apreciar esta concentración de talento y energía, pero al mismo tiempo, estos beneficios urbanos representan una pérdida trágica para las regiones de las cuales vienen los migrantes, posponiendo indefinidamente sus propias posibilidades de desarrollo. En el Chocó casi no hay entre los habitantes conciencia del costo que implica para la sociedad su propia movilidad social y educativa. Todos los adolescentes son altamente conscientes del 
subdesarrollo del Chocó, pero la única respuesta que tienen es la de permanecer en la escuela. Mientras que puedan salir avantes en el logro de algunas aspiraciones personales, su escolaridad tiene un efecto negativo abrumador en la posibilidad del desarrollo chocoano.

De ahí que la educación llegue a ser solamente una manera más con la cual el "exterior" oprime a los chocoanos extrayendo su juventud más enérgica mientras que dejan a la región en el atraso.

Traducción del inglés por Leonor Cuervo de Rojas y Priscila Torres Rodríguez

Técnicos Centro de Investigaciones de la Universidad Pedagógica Nacional 